\title{
Stepwise metabolic engineering of Candida tropicalis for efficient xylitol production from xylose mother liquor
}

\author{
Lihua Zhang, Zhen Chen, Junhua Wang, Wei Shen, Qi Li and Xianzhong Chen ${ }^{*}$ (1)
}

\begin{abstract}
Background: Commercial xylose purification produces xylose mother liquor (XML) as a major byproduct, which has become an inexpensive and abundant carbon source. A portion of this XML has been used to produce low-valueadded products such as caramel but the remainder often ends up as an organic pollutant. This has become an issue of industrial concern. In this study, a uracil-deficient Candida tropicalis strain was engineered to efficiently convert XML to the commercially useful product xylitol.

Results: The xylitol dehydrogenase gene was deleted to block the conversion of xylitol to xylulose. Then, an NADPH regeneration system was added through heterologous expression of the Yarrowia lipolytica genes encoding 6-phosphate-gluconic acid dehydrogenase and 6-phosphate-glucose dehydrogenase. After process optimization, the engineered strain, C. tropicalis XZX-B4ZG, produced $97.10 \mathrm{~g} \mathrm{~L}^{-1}$ xylitol in $120 \mathrm{~h}$ from $300 \mathrm{~g} \mathrm{~L}^{-1}$ XML in a 5-L fermenter. The xylitol production rate was $0.82 \mathrm{~g} \mathrm{~L}^{-1} \mathrm{~h}^{-1}$ and the conversion rate was $92.40 \%$.
\end{abstract}

Conclusions: In conclusion, this study performed a combination of metabolic engineering and process optimizing in C. tropicalis to enhance xylitol production from XML. The use of C. tropicalis XZX-B4ZG, therefore, provided a convenient method to transform the industrial by-product XML into the useful material xylitol.

Keywords: Xylose mother liquor, Candida tropicalis, Cofactor regeneration, Xylitol, Fermentation optimization

\section{Background}

Xylitol is a five-carbon sugar alcohol widely and increasingly used in food, medicines and the chemical industry. It is currently produced on an industrial scale via the catalytic hydrogenation of D-xylose [1]. The xylose used in this process is generally extracted from the acid hydrolysate of hemicellulose obtained from sources such as corncob and sugarcane bagasse [2]. This acid hydrolysate contains many other sugars and impurities that complicate xylose purification and lead to the production of xylose mother liquor (XML) as the major by-product of

*Correspondence: liqi@jiangnan.edu.cn; xzchen@jiangnan.edu.cn Key Laboratory of Industrial Biotechnology, Ministry of Education, School of Biotechnology, Jiangnan University, 1800 Lihu Road, Wuxi 214122, People's Republic of China xylose manufacture $[3,4]$. XML is a dark brown, nutrient-dense liquor that contains xylose (35-40\%), glucose (8-10\%), L-arabinose (10-15\%), D-galactose (8-10\%), and trace amount of other sugars $[1,5,6]$. The production of one ton of xylose results in the formation of one to one and a half tons of XML. It has been estimated that several large factories in China produce $50,000-80,000$ tons of XML per year [1]. A portion of this XML has been used to produce low-value-added products such as caramel but the remainder often ends up as an organic pollutant. This has become an issue of industrial concern [7].

A common strategy to avoid pollution and improve the economic value of XML is to isolate the individual sugars using simulated moving-bed chromatography. However, the current techniques for sugar separation are inefficient, and the operating costs are high compared with 
the value of the sugars $[1,8]$. An alternate strategy is to increase the proportion of the sugar of interest by removing other sugars through microbial fermentation. For example, Cheng et al. isolated a yeast strain that could metabolize most of the sugars, but not L-arabinose [8]. Use of this strain increased the L-arabinose content of the XML to $86.1 \%$ of total sugars, allowing the L-arabinose to be isolated using a simple process. Other microbial processes have been used to convert XML to high-value chemicals such as xylitol $[1,6]$, solvents [3, 9], welan gum [10] and succinic acid [7]. However, XML also contains furfural and 5-hydroxymethyl furfural, which are potent inhibitors of microbial growth. Furfurals cause the accumulation of reactive oxygen species and cellular damage in Saccharomyces cerevisiae [11] and increase the toxicity of phenols in Escherichia coli [12]. Furfurals also inhibit xylitol production from non-detoxified corncob hemicellulose acid hydrolysate [13]. Therefore, XML must be detoxified before it can be used as a fermentation feedstock. Unfortunately, the processes used to detoxify XML strip it of nutrients and increase production costs. In our opinion, there is an urgent need for simpler biotechnological systems that can improve the value of XML.

One potential solution that addresses the problem of XML accumulation through microbial fermentation while mitigating the need to detoxify the XML is the use of Candida tropicalis, a diploid yeast, to convert XML to xylitol [14]. This conversion is catalyzed by the enzyme xylose reductase, which requires NADPH as a coenzyme. Unfortunately, C. tropicalis also expresses the enzyme xylitol dehydrogenase (XDH), which prevents xylitol accumulation by oxidizing it to xylulose (Fig. 1). Disruption of the $X Y L 2$ genes, which encode $\mathrm{XDH}$, has been shown to increase the xylitol yield to $98 \%$, with a volumetric productivity of $3.23 \mathrm{~g}^{-1} \mathrm{~L}^{-1} \mathrm{~h}^{-1}$, when pure xylose was used as the substrate [15]. The availability of $\mathrm{NADPH}$ is also a major limitation, as volumetric xylitol productivity was higher when NADPH regeneration was enhanced in a defined medium [16, 17]. XML was recently used as a feedstock for xylitol production by a combination of two complementary strains: wild-type $C$. tropicalis and xylA-disrupted Bacillus subtilis [1]. A conversion rate of $0.75 \mathrm{~g} \mathrm{~g}^{-1}$ was obtained. In that one-pot system, C. tropicalis was used both to deplete furfural and 5-hydroxymethyl furfural and to convert xylose into xylitol. Research focusing on the mechanisms of furfural tolerance has shown that NADH formation during xylose metabolism (Fig. 1) facilitates the detoxification of furfural, and that the half maximal inhibitory concentration of furfural for C. tropicalis was much higher $\left(3.69 \mathrm{~g} \mathrm{~L}^{-1}\right)$ than that of other microbes [18]. This enhanced furfural tolerance enhances the ability of $C$. tropicalis to process $\mathrm{XML}$ and other biomass hydrolysates.

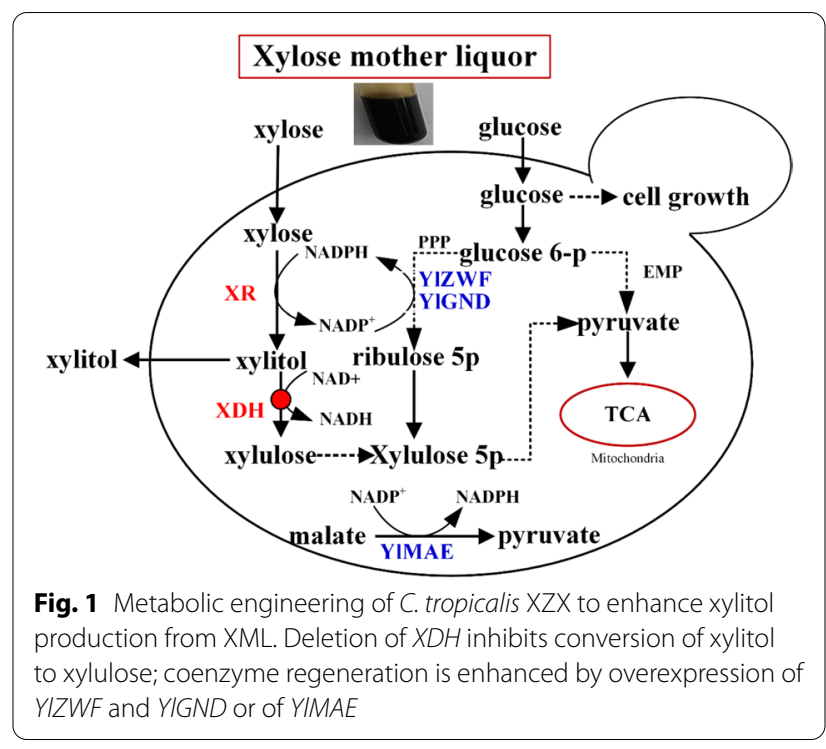

In a previous study, we developed an efficient strategy for the genetic manipulation of $C$. tropicalis through sequential gene disruption and expression [19]. In this study, the xylose metabolism network of $C$. tropicalis was rationally modified to facilitate xylitol production using $\mathrm{XML}$ as the fermentation feedstock. Firstly, the $X Y L 2$ genes of $C$. tropicalis strain XZX were disrupted to block the conversion of xylitol to xylulose. Secondly, two parallel NADPH coenzyme regeneration systems were constructed, one involving the pentose phosphate pathway and the other involving malate formation. Subsequent testing and process optimization yielded a simple and efficient process to produce xylitol from XML with high productivity.

\section{Results}

\section{Analysis of the XML used in this study}

The chemical composition of XML varies widely depending on the corn cob hydrolysis and crystallization processes used during xylose production [1]. Therefore, the composition of the XML used in this study was characterized. After being sterilized in an autoclave, the XML was found to contain $98.36 \mathrm{~g} \mathrm{~L}^{-1}$ glucose, $512.31 \mathrm{~g} \mathrm{~L}^{-1}$ xylose and $96.28 \mathrm{~g} \mathrm{~L}^{-1}$ arabinose. Furfural, acetate and phenol were also detected. The acetate and phenol concentrations were very low, but the furan compounds (furfural and 5-hydroxymethyl furfural) concentration in XML was $7.3 \mathrm{~g} \mathrm{~L}^{-1}$ (of which furfural was $4.2 \mathrm{~g} \mathrm{~L}^{-1}$ ). The effect of compounds such as furfural and acetate on the growth of C. tropicalis cells was investigated using $200 \mathrm{~g}$ $\mathrm{L}^{-1}(\mathrm{w} / \mathrm{v})$ XML as the carbon source (furfural $0.82 \mathrm{~g}$ $\mathrm{L}^{-1}$, 5-hydroxymethyl furfural $0.65 \mathrm{~g} \mathrm{~L}^{-1}$ ). Although reports have stated that furfural can seriously suppress 
the growth of many microorganisms $[13,18]$, we found that $C$. tropicalis XZX could grow at a rapid rate (see Additional file 1: Fig. S1), consistent with previous studies showing that $C$. tropicalis is tolerance to these compounds [18]. These results suggested that $C$. tropicalis XZX could be transformed into a useful host for xylitol production from XML.

\section{Characterization of $X Y L 2$-disrupted strain}

For C. tropicalis XZX to accumulate useful quantities of xylitol, xylitol dehydrogenase activity must be eliminated through gene deletion (Fig. 1). The two XYL2 alleles were sequentially deleted using the established genetic manipulation strategy [19]. A series of mutant strains were constructed during this process, including $C$. tropicalis XZX-B1 (ura3/ura3 xyl2::gda-URA3/XYL2), C. tropicalis XZX-B2 (ura3/ura3 xyl2::gda/XYL2), C. tropicalis XZXB3 (ura3/ura3 xyl2::gda/xyl2::gda-URA3) and C. tropicalis XZX-B4 (ura3/ura3 xyl2::gda/xyl2::gda).

C. tropicalis strains XZX, XZX-B2 and XZX-B4 were cultured at $30{ }^{\circ} \mathrm{C}$ for 3 days on $\mathrm{SM}$ plates containing xylose as the sole carbon source. Strains XZX (control) and XZX-B2 grew well, but XZX-B4 was unable to grow (see Additional file 1: Fig. S2). The control and XZX-B2 exhibited similar growth rates and final cell concentrations in liquid SM containing xylose as the sole carbon source. However, the growth of XZX-B4 was completely inhibited by the deletion of both $X Y L 2$ allelic genes (Fig. 2b). In contrast, deletion of both $X Y L 2$ alleles had only a slight impact on growth when glucose was used as the sole carbon source (Fig. 2a). Thus, deleting one XYL2 gene had no effect on xylose utilization and cell growth as the other $X Y L 2$ gene could complement xylose metabolism. However, deletion of both $X Y L 2$ genes eliminated the cell's ability to grow when using xylose as the sole carbon source.

In our previous study, the xylitol accumulation of $X Y L 2$-disrupted strains was evaluated using a mixture of glucose and xylose as the carbon source (Fig. 2c, d, f) [20]. Strains XZX and XZX-B2 grew at a rate higher than XZX-B4 (Fig. 2c). They continued to grow, using xylose as the carbon source, when glucose was depleted (Fig. 2d, e). In contrast, XZX-B4 stopped growing after glucose was depleted and yielded a lower final cell mass (Fig. 2c, d). Furthermore, the xylitol titer of XZX-B2 was higher than those of XZX and XZX-B4 (Fig. 2f). Interestingly, the conversion rate (xylitol titer versus xylose consumed) of XZX-B4 reached $99 \%$, which is near the theoretical value $(100 \%)$ and much higher than those of the other two strains (Fig. 2d, f).

\section{Increasing coenzyme regeneration in C. tropicalis}

Since xylose reductase uses NADPH preferentially or exclusively as a coenzyme [21, 22] (Fig. 1), the low xylitol
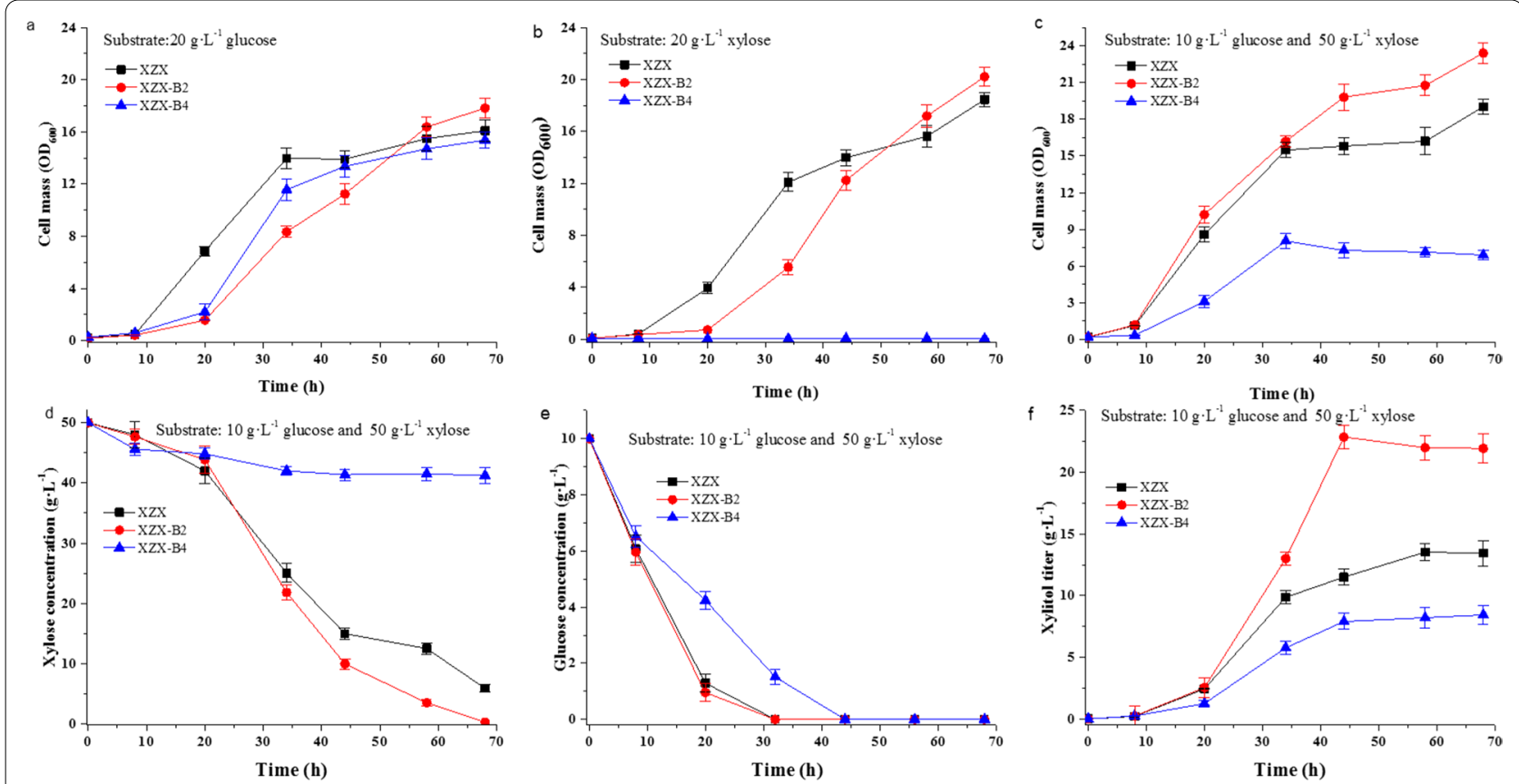

Fig. 2 Characterization of strains XZX-B2, XZX-B4 and control strain XZX. Growth patterns were evaluated using: a glucose, b xylose, or c a mixture of glucose and xylose as the carbon source. Evaluation of (d) xylose consumption, (e) glucose consumption, and (f) xylitol titer when using a mixture of glucose and xylose as the carbon source. Error bars indicate standard deviations from the mean of triplicate biological replicates 
titer observed with XZX-B4 may have been caused by a low intracellular NADPH concentration. To test this hypothesis, two different coenzyme regeneration systems were created and their impact on xylitol production and cell growth were investigated.

Since the pentose phosphate pathway is the primary means of NADPH generation, diversion of glycolytic flux from the Embden-Meyerhof-Parnas pathway to the pentose phosphate pathway should increase NADPH availability [23]. Therefore, two critical enzymes of the pentose phosphate pathway (glucose 6-phosphate dehydrogenase and 6-phosphogluconate dehydrogenase) were overexpressed (Fig. 1). To accomplish this, YlZWF and YlGND from the non-conventional yeast Yarrowia lipolytica were incorporated into separate expression cassettes that were inserted into XZX-B4 in a two-step procedure. First, the cassette containing YlZWF was used to transform XZX-B4, creating C. tropicalis XZX-B4Z. PCR (see Additional file 1: Fig. S3b) and DNA sequencing were used to confirm the genomic modification. An assessment of gene expression using $\mathrm{qPCR}$ indicated that $Y l Z W F$ was being expressed (see Additional file 1: Fig. S4a). In the second step, the cassette containing YlGND was used to transform XZX-B4Zt, creating C. tropicalis XZX-B4ZG. This genomic modification was also confirmed using PCR (see Additional file 1: Fig. S3c), and YlGND expression was confirmed using qPCR (see Additional file 1: Fig. S4b). Meanwhile, the YlGND expression cassette was transformed into XZX-B, creating C. tropicalis XZX-B4G.

In the second NADPH regeneration system, malic enzyme was overexpressed. Malic enzyme converts the tricarboxylic acid cycle intermediate malate into pyruvate while simultaneously converting $\mathrm{NADP}^{+}$to NADPH [24]. To accomplish this, YlMAE from Y. lipolytica CICC 31,251 was inserted into an expression cassette that was used to transform XZX-B4, creating C. tropicalis XZXB4M. The genomic modification was confirmed by PCR (see Additional file 1: Fig. S3d) and DNA sequencing, and YIMAE expression was confirmed using qPCR (see Additional file 1: Fig. S4c).

Strains XZX-B3, XZX-B4Z, XZX-B4ZG and XZX-B4M to assess the impact of the NADPH regeneration systems on cell growth and xylitol production. Strain XZX-B3 was used as the control, rather than strain XZX-B4, because strain XZX-B3 exhibits the $\mathrm{ura}^{+}$phenotype (Table 1)

Table 1 Strains and plasmids used in this study

\begin{tabular}{|c|c|c|}
\hline & Description & Reference or source ${ }^{a}$ \\
\hline \multicolumn{3}{|l|}{ Strains } \\
\hline E. coli JM109 & $\begin{array}{l}\text { recA1 endA1 gyrA96 thi-1 hsdRI7 }\left(r^{-} \mathrm{mk}^{+}\right) \text {e14- }\left(\mathrm{mcrA}^{-}\right) \text {supE44 relA1 } \triangle \text { (lac- } \\
\text { proAB)/F' } \mathrm{F}^{\prime}\left[\text { traD36 proAB }{ }^{+} \text {laclq lacZ } \triangle \mathrm{M} 15\right]\end{array}$ & TaKaRa \\
\hline C. tropicalis ATCC 20,336 & URA3/URA3 XYL2/XYL2 & ATCC \\
\hline Y. lipolytica CICC 31,251 & & $\mathrm{CICC}$ \\
\hline C. tropicalis XZX & ura3/ura3 XYL2/XYL2 & [19] \\
\hline C. tropicalis XZX-B1 & ura3/ura3 xyl2::gda-URA3/XYL2 & This study \\
\hline C. tropicalis XZX-B2 & ura3/ura3 xyl2::gda/XYL2 & This study \\
\hline C. tropicalis XZX-B3 & ura3/ura3 xyl2::gda/xyl2::gda-URA3 & This study \\
\hline C. tropicalis XZX-B4 & ura3/ura3 xy/2::gda/xy/2::gda & This study \\
\hline C. tropicalis XZX-B4G & $\begin{array}{l}\text { ura3/ura3 xyl2::gda-URA3-P } \text { GAPDH }^{-G N D-T_{G A P D H}} / \\
\text { xyl2::gda }\end{array}$ & This study \\
\hline C. tropicalis XZX-B4Z & $\begin{array}{l}\text { ura3/ura3 xyl2::gda-URA3-P } P_{G A P D H}-Z W F-T_{G A P D H} / \\
\text { xyl2::gda }\end{array}$ & This study \\
\hline C. tropicalis XZX-B4Zt & ura3/ura3 xyl2::gda- $P_{\text {GAPDH }}-Z W F-T_{G A P D H} / x y \mid 2::$ gda & This study \\
\hline C. tropicalis XZX-B4ZG & ura3/ura3 xyl2::gda- $P_{G A P D H}-Z W F-T_{G A P D H} / x y \mid 2:: g d a-U R A 3-P_{G A P D H}-G N D-T_{G A P D H}$ & This study \\
\hline C. tropicalis XZX-B4M & $\begin{array}{l}\text { ura3/ura3 xyl2::gda-URA3-P } P_{G A P D H}-M A E-T_{G A P D H} \\
\text { /xyl2::gda }\end{array}$ & This study \\
\hline \multicolumn{3}{|l|}{ Plasmids } \\
\hline Tm-gda-URA3 & Contain a functional marker gene flanked by gda & [19] \\
\hline Ts-XYL2I-gda-URA3 & Contain the cassette for the disruption of the first XYL2 allele & This study \\
\hline Ts-XYL2II-gda-URA3 & Contain the cassette for the disruption of the second XYL2 allele & This study \\
\hline Ts-XYL2l-gda-URA3-P $P_{G A P D H}-Y I Z W F-T_{G A P D H} \cdot$ & Contain the YIZWF gene expressioncassette & This study \\
\hline Ts-XYL2II-gda-URA3-P ${ }_{G A P D H}-Y I G N D-T$ GAPDH & Contain the YIGND gene expression cassette & This study \\
\hline Ts-XYL2I-gda-URA3-P GAPDH $-Y I M A E-T_{G A P D H}$ & Contain the YIMAE gene expression cassette & This study \\
\hline
\end{tabular}

${ }^{a}$ ATCC: American Type Culture Collection; CICC: China Center of Industrial Culture Collection 
and displays better growth performance than XZX-B4, which is $\mathrm{ura}^{-}$. The four strains were grown in fermentation medium (a mixture of $10 \mathrm{~g} \mathrm{~L}^{-1}$ glucose and $50 \mathrm{~g} \mathrm{~L}^{-1}$ xylose as the carbon source) and cell growth, xylose consumption, xylitol production, and intracellular NADPH concentration were evaluated. Strain XZX-B4ZG produced the highest cell mass and consumed the most xylose (Fig. 3a, b), producing xylitol with a titer of $17.5 \mathrm{~g}$ $\mathrm{L}^{-1}$ with a bioconversion rate of $95.1 \%$ (Fig. 3c). Interestingly, XZX-B4Z produced higher cell mass, xylose consumption and xylitol titer than XZX-B3 (Fig. 3). And the lag phase of XZX-B4Z and XZX-B4ZG was significantly reduced when growing in fermentation medium with $2 \mathrm{~g} \mathrm{~L}^{-1}$ of furfural (see Additional file 1: Fig. S5). Previous studies demonstrated that overexpression of $Z W F 1$ (which encodes glucose 6-phosphate dehydrogenase) in $S$. cerevisiae allowed growth at furfural concentrations that are normally toxic, whereas a $z w f 1^{-}$mutant displayed higher furfural sensitivity [25]. Our results led to a similar conclusion. Overexpression of YlZWF in $C$. tropicalis could play a similar role while improving xylitol production.

\section{Effect of fermentation conditions on xylitol production}

In initial experiments using $100 \mathrm{~g} \mathrm{~L}^{-1} \mathrm{XML}$ as the substrate, strain XZX-B4ZG produced the highest xylitol titer $\left(25.9 \pm 1.34 \mathrm{~g} \mathrm{~L}^{-1}\right)$, with a yield of $87.8 \%(\mathrm{~mol} / \mathrm{mol}$; see Additional file 1: Table S1). Thus, introduction of this NADPH regeneration system had a significant impact on xylitol production from XML, supporting our hypothesis that supplying sufficient coenzyme could improve xylitol production. Despite this achievement, this xylitol titer was still lower than that desired for industrial-scale xylitol production. To meet this goal, xylitol production by XZX-B4ZG was optimized.

The effect of initial $\mathrm{pH}$ was evaluated using $\mathrm{pH}$ values from 4 to 7 (Fig. 4a). Cell mass increased with $\mathrm{pH}$ value over this range, but the xylitol titer $\left(29.3 \mathrm{~g} \mathrm{~L}^{-1}\right)$ and conversion rate $(93.5 \%)$ peaked at $\mathrm{pH} 5.0$, so $\mathrm{pH} 5.0$ was used in subsequent fermentations. A previous study showed that the optimal temperatures for cell growth and desired product synthesis were different [26]. In this study, the effect of temperature on xylitol production by XZXB4ZG was evaluated at $30,33,35$ and $37{ }^{\circ} \mathrm{C}$ (Fig. 4b). Consistent with the previous study, cell mass tended to decrease with increasing temperature, but xylitol titer and yield both peaked at $35{ }^{\circ} \mathrm{C}$. At the optimal temperature, the xylitol titer was $29.9 \mathrm{~g} \mathrm{~L}^{-1}$ xylitol with a yield of $93.3 \%$. Therefore, subsequent fermentations were performed at $35^{\circ} \mathrm{C}$.

Having optimized $\mathrm{pH}$ and temperature, additional experiments were conducted to investigate the effects of $\mathrm{XML}$ concentration on cell growth, xylose consumption
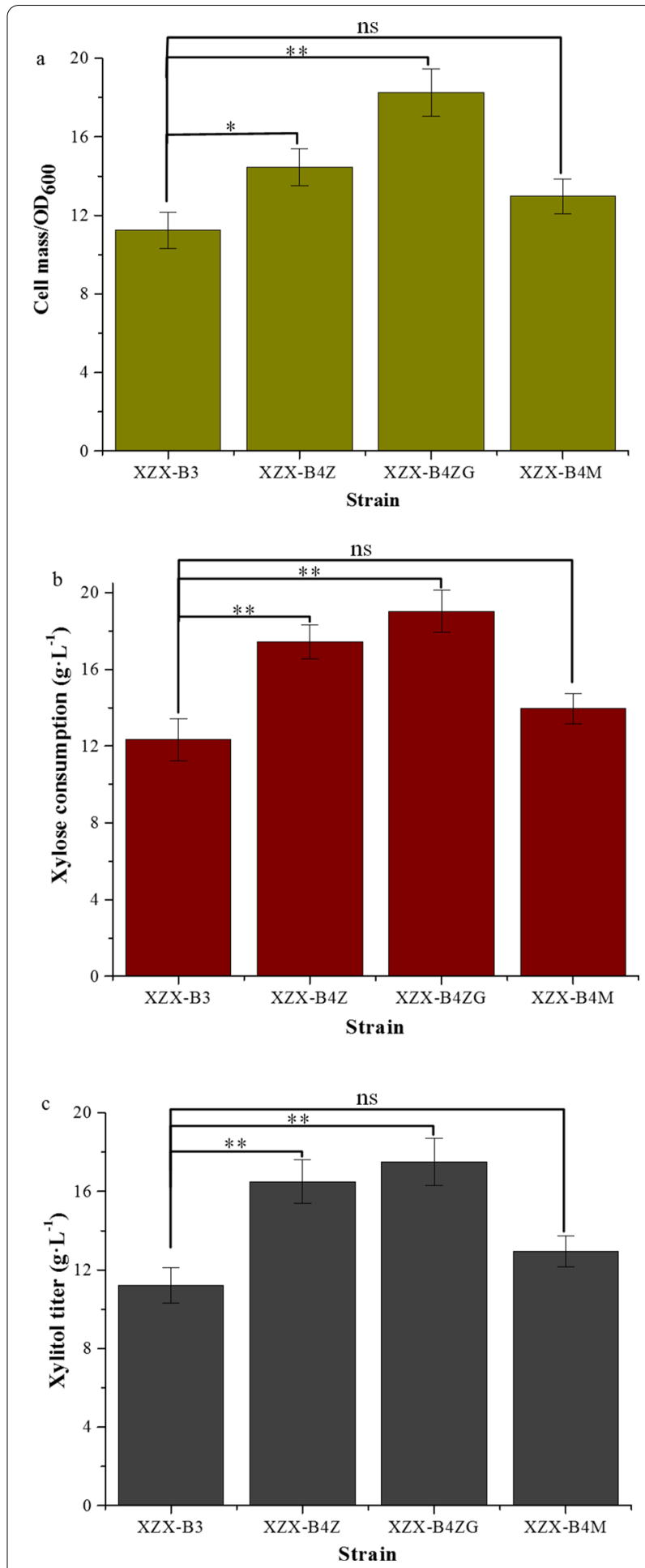

Fig. 3 Effect of coenzyme regeneration systems. Comparisons of: a cell mass, $\mathbf{b}$ xylose consumption, and (c) xylitol titer among C. tropicalis strains XZX-B4Z, which expresses YIZWF; ZX-B4ZG, which overexpresses both YIZWF and YIGND; and XZX-B4M, which overexpresses YIMAE. The control strain, XZX-B3 is the precursor of parent $\mathrm{XZX}-\mathrm{B} 4$ that retains the URA3 marker. Error bars indicate standard deviations from the means of triplicate biological replicates. Differences were analyzed by Student's $t$-test. ${ }^{* *} p<0.01$, * $p<0.05$; "ns" means not significant 

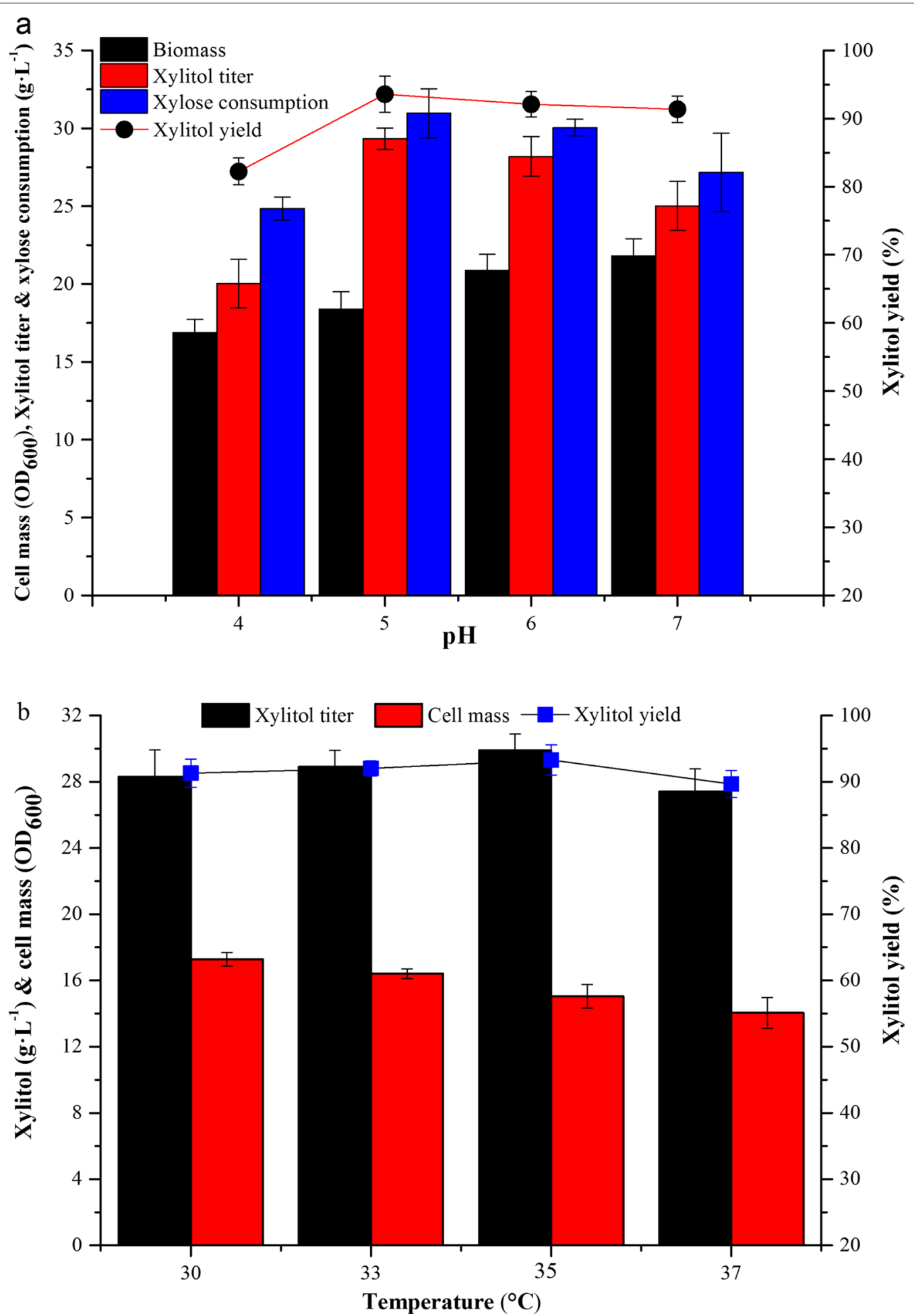

Fig. 4 Optimization of fermentation conditions for xylitol production from XML by C. tropicalis XZX-B4ZG. a Effect of pH on biomass, xylose consumption, xylitol titer and xylitol yield. $\mathbf{b}$ Effect of temperature on biomass, xylitol titer and xylitol yield. Fermentations were conducted in shake flasks containing $100 \mathrm{~g} \mathrm{~L}^{-1}$ XML. Error bars indicate standard deviations from the means of triplicate biological replicates 
and xylitol production (Fig. 5). Cells grew rapidly and produced a relatively high xylitol yield at early times when the XML concentration was $100-150 \mathrm{~g} \mathrm{~L}^{-1}$, but the cell mass began to decline after $24 \mathrm{~h}$ (Fig. 5a) due to glucose exhaustion (data not shown). After $96 \mathrm{~h}$, the xylitol titer was significantly lower than that obtained using higher XML concentrations. Higher XML concentrations (200-300 $\mathrm{g} \mathrm{L}^{-1}$ ) produced higher xylitol titers, but the higher XML concentrations also hampered cell growth, probably because of the increased concentrations of inhibitors in the fermentation medium. Despite the slower growth rates, the final cell masses were similar to those obtained using lower XML concentrations, suggesting that strain XZX-B4ZG could adapt to this stress during fermentation. After 96 h, XZX-B4ZG cultured in fermentation medium containing XML $250 \mathrm{~g} \mathrm{~L}^{-1}$ produced $58.9 \mathrm{~g} \mathrm{~L}^{-1}$ xylitol with a yield of $98 \%$. Therefore, $250 \mathrm{~g} \mathrm{~L}^{-1}$ was used as the optimal XML concentration for xylitol bioconversion.

Yeast extract, peptone and corn steep liquor were investigated as alternate nitrogen sources. The results showed that the lack of nitrogen sources seriously inhibited cell growth, but changing the nitrogen sources had minimal impacts on cell growth, xylose consumption rate, and xylitol titer (see Additional file 1: Fig. S6). Corn steep liquor seemed the best choice because its use led to the highest xylitol titer $\left(53.6 \mathrm{~g} \mathrm{~L}^{-1}\right)$ with a yield of $95.22 \%$ and because of its low cost.

\section{Enhanced production of xylitol from XML in a 5-L bioreactor}

To better assess the potential of strain XZX-B4ZG to produce xylitol from XML on an industrial scale, fermentation was performed in a 5-L bioreactor. Using conditions obtained from the optimization experiments, the cell mass peaked at $\sim 16 \mathrm{~h}$ (Fig. 6a), which was much earlier than that observed during shake-flask cultivation. Glucose was depleted at a correspondingly fast rate (Fig. 6a). The rapid initial growth rate led to a significant decrease in dissolved oxygen (DO) and the $\mathrm{pH}$ displayed a similar variation (Fig. 6b). When glucose was depleted and xylitol had begun to accumulate, the DO began to increase slowly (Fig. 6b). To generate reducing force NADPH for xylose reduction, glucose was added as co-substrate when its concentration was lower than $1 \mathrm{~g}$ $\mathrm{L}^{-1}$. The xylitol accumulation rate increased after $8 \mathrm{~h}$, leading to a final xylitol concentration of $97.1 \mathrm{~g} \mathrm{~L}^{-1}$ with a productivity of $0.82 \mathrm{~g} \mathrm{~L}^{-1} \mathrm{~h}^{-1}$ (Fig. 6c). These results suggest that fermentation of $C$. tropicalis XZX-B4ZG in a medium containing XML constitutes an efficient and simple bioprocess for xylitol production from XML, a low-cost biomass resource.
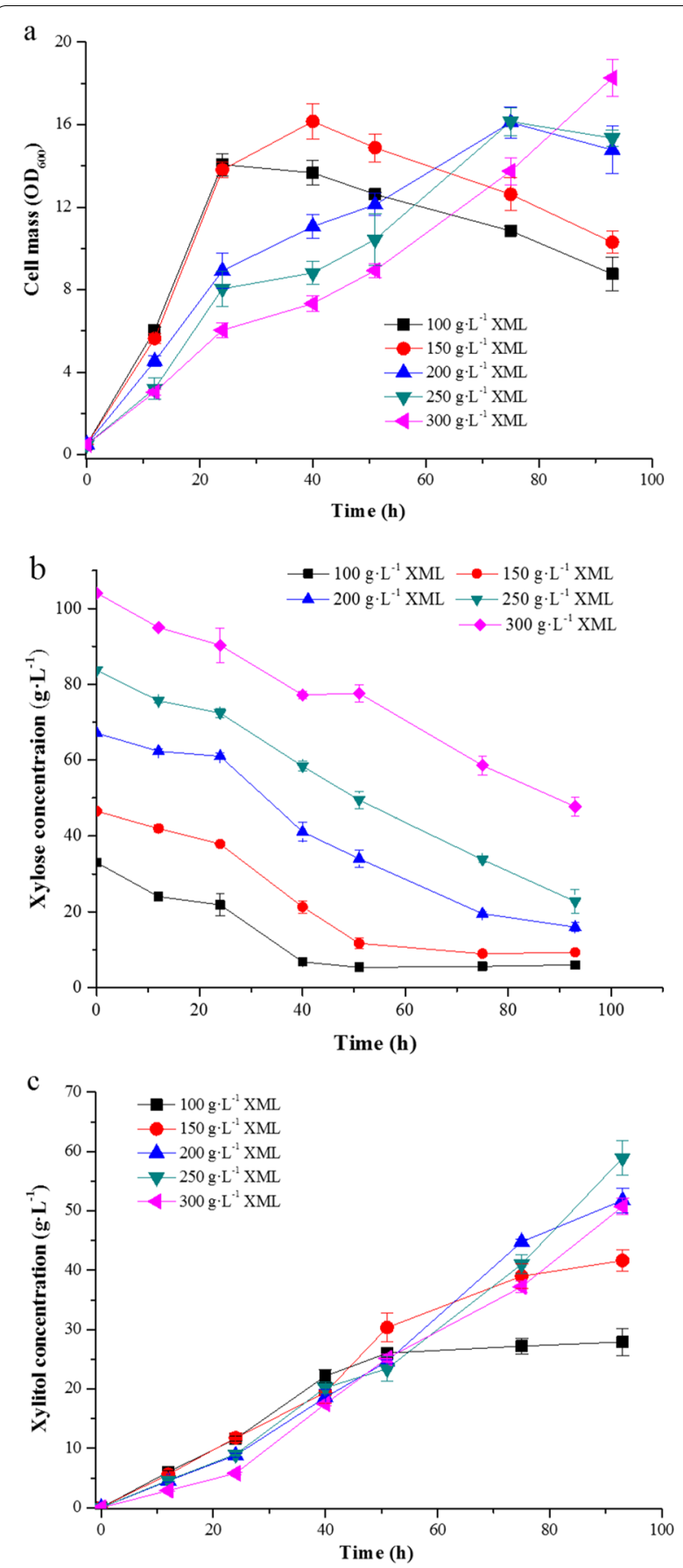

Fig. 5 Effect of XML concentration on the fermentation process. a Effect on cell growth. $\mathbf{b}$ Effect on xylose concentration. $\mathbf{c}$ Effect on xylitol concentration. Error bars indicate standard deviations from the means of triplicate biological replicates

\section{Discussion}

The xylose mother liquor is an abundant biomass byproduct and a potential environmental pollutant in China. Here, we sought to apply metabolic engineering 

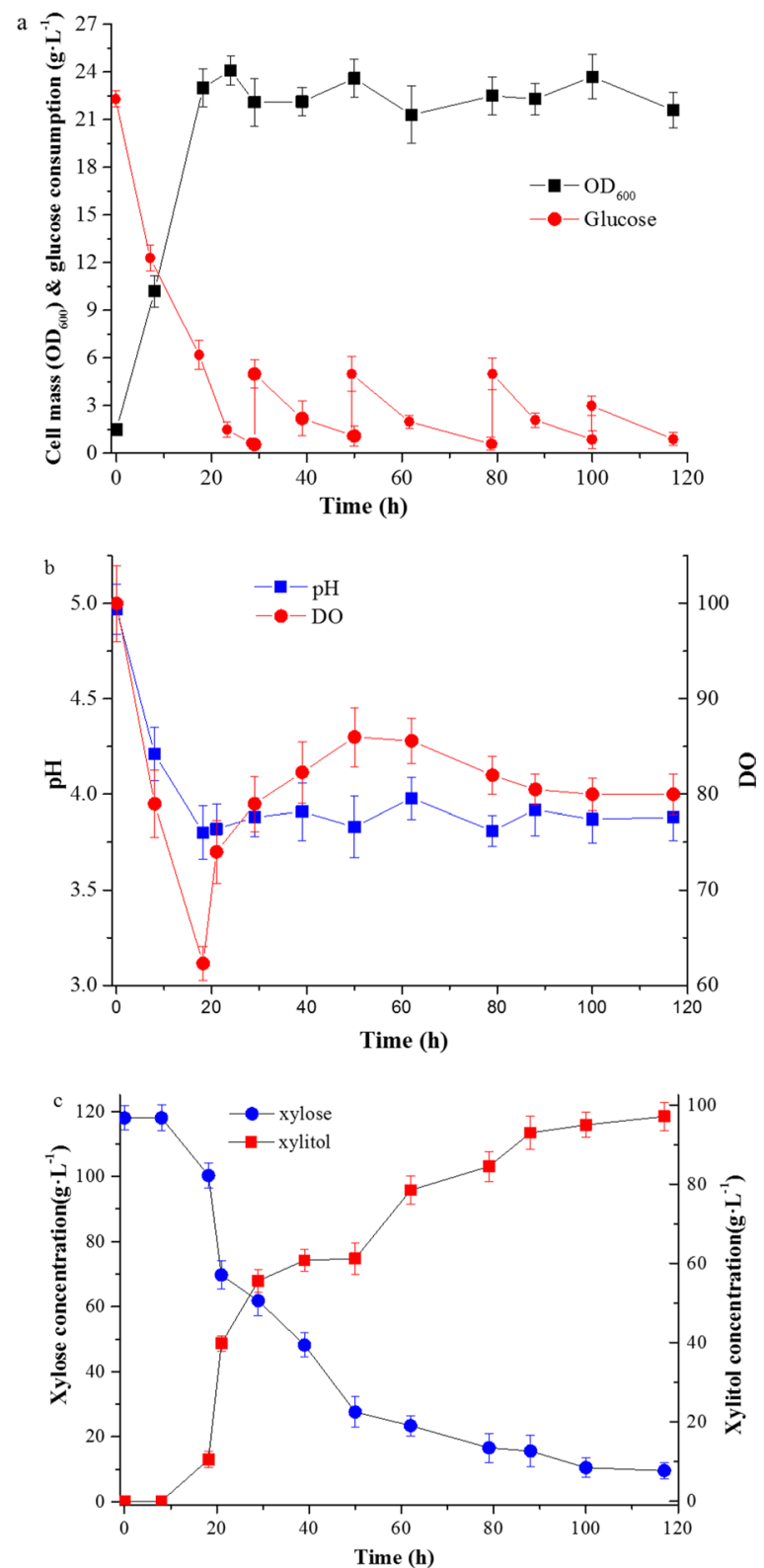

Fig. 6 Xylitol production from XML in a 5-L fermenter. Representative result obtained at $35^{\circ} \mathrm{C}$ with stirring at $700 \mathrm{r} \mathrm{min}^{-1}$ and a aeration rate $4 \mathrm{v} \mathrm{v}^{-1}$.min. a Cell mass growth and glucose consumption profiles, glucose was fed when concentration was below $1 \mathrm{~g} / \mathrm{L}$. b pH and dissolved oxygen (DO) profiles. $\mathbf{c} x y$ litol accumulation and xylose consumption profiles. Error bars indicate standard deviations from the means of triplicate biological replicates

to industrial yeast strain for improving XML value. Genetic manipulations combined cofactor regeneration and process optimization resulted in engineered $C$. tropicalis efficiently overproducing xylitol from XML.

To improve the productivity of xylitol, two different coenzyme regeneration systems were created in
Table 2 NADPH/NADP + contents of strains described in this study

\begin{tabular}{llllr}
\hline Strains & $\begin{array}{l}\text { NADPt } \\
\text { (pmol/ } \\
\text { well) }\end{array}$ & $\begin{array}{l}\text { NADPH } \\
\text { (pmol/ } \\
\text { well) }\end{array}$ & $\begin{array}{l}\text { NADP }^{+} \\
\text {(pmol/ } \\
\text { well) }\end{array}$ & NADPH/NADP $^{+}$ \\
\hline XZX-B3 & 24.53 & 22.52 & 2.01 & 11.20 \\
XZX-B4Z & 34.52 & 29.82 & 4.72 & 6.32 \\
XZX-B4G & 30.87 & 28.14 & 2.73 & 10.31 \\
XZX-B4ZG & 38.50 & 30.62 & 7.88 & 3.86 \\
XZX-B4M & 32.37 & 28.63 & 3.74 & 7.70 \\
\hline
\end{tabular}

$\mathrm{NADPt}$, total of $\mathrm{NADP}^{+}$and $\mathrm{NADPH}$ concentrations

C. tropicalis. Simultaneous expression of $Y l Z W F$ and YlGND (strain XZX-B4ZG) significantly increased intracellular NADPH availability (Table 2), which was approximately 1.36-fold higher than that of XZX-B3. In contrast, individual expression of YlZWF (XZX-B4Z), YlGND (XZX-B4G) or YlMAE (XZX-B4M) only slightly increased intracellular NADPH availability (Table 2). Clearly, the reactions catalyzed by YlZWF and YlGND both limit flux through the pentose phosphate pathway, so that overexpression of each alone cannot substantially increase intracellular NADPH availability. Interestingly, all of the yeast strains containing augmented NADPH regeneration systems accumulated high amounts of NADP+. Previous studies have demonstrated cytosolic NADPH/NADP + ratios ranging from 15 to 60 . These ratios drive the biosynthesis of fatty acids and nucleic acids, as well as the defense against oxidative stress $[27,28]$. Our results revealed that overexpressing YlZWF, YlGND and/or YlMAE slightly decreased the NADPH/NADP ${ }^{+}$ratio, even though the intracellular NADPH supply increased. Further research is needed because augmentation of the pentose phosphate pathway is expected to increase the $\mathrm{NADPH} / \mathrm{NADP}^{+}$ratio [29].

As shown in Fig. 2d, e and C. tropicalis utilized the glucose preferentially over xylose (the so-called carbon catabolite repression) in the medium containing a mixture carbon sources. The similar phenomenon was reported in Kluyveromyces marxianus [30] and S. cerevisiae [31]. This increased the difficulty for fermentation control and further limited xylitol productivity. Because xylose can not be used for growing of the engineered strain C. tropicalis XZX-B4ZG. Once the glucose was exhausted, xylitol accumulation is stopped due to lack of coenzyme for xylose reduction. To tackle this problem, the endogenous KmHXK1 gene (encoding hexokinase 1 which involved in the glucose repression) in K. marxianus was knocked out and the exogenous xylose-specific transporter gene was overexpressed 
[30]. The engineered $K$. marxianus strain could co-utilize the xylose and glucose in fermentation medium for xylitol production. Construction of chimeric C. tropicalis-recombinant Bacillus subtilis co-cultures enabled high efficiency production of xylitol and the by-products in XML was used up as well, by taking advantage of combination the arabinose and galactose metabolism in Bacillus subtilis with high-efficiency xylitol biosynthesis in C. tropicalis [1]. Although the one-pot biotransformation has some unique advantages, constructing a "perfect" strain by metabolic engineering for monoculture is always attractive. In a previous report, the bacteria arabinose metabolic pathway was introduced into XYL2-disrupted C. tropicalis [32]. The resulting recombinant strain converted xylose into xylitol, without arabitol formation. Perhaps this strategy for removing arabinose from fermentation medium could also be taken for transforming XML into xylitol in largely industrial-scale production.

\section{Conclusions}

In summary, C. tropicalis was engineered systematically to address the bottlenecks that sequentially limit xylitol biosynthesis from xylose. Genetic manipulations combined cofactor regeneration and process optimization resulted in engineered $C$. tropicalis efficiently overproducing xylitol from XML. Under the optimized conditions, the engineered strain, C. tropicalis XZXB4ZG, produced $97.10 \mathrm{~g} \mathrm{~L}^{-1}$ xylitol with $92.40 \%$ conversion rate from $300 \mathrm{~g} \mathrm{~L}^{-1}$ xylose mother liquor in a $5-\mathrm{L}$ fermenter. The stepwise engineering strategy, as well as process investigation, can be used to rationally design cell factories for the eco-friendly use of xylose mother liquor and the production of highly valuable chemicals.

\section{Materials and methods}

\section{Strains, media and culture conditions}

The strains and plasmids used in this work are listed in Table 1. C. tropicalis XZX, a uracil auxotrophic derivative of C. tropicalis ATCC 20,336, was used as the parent strain for genetic manipulation [19]. Minimal mediun (MM; $6.7 \mathrm{~g} \mathrm{~L}^{-1}$ yeast nitrogen base, $20 \mathrm{~g} \mathrm{~L}^{-1}$ glucose and $\left.10 \mathrm{~g} \mathrm{~L}^{-1}\left(\mathrm{NH}_{4}\right)_{2} \mathrm{SO}_{4}\right)$ and supplemented medium (SM; MM supplemented with uracil at a concentration of $0.06 \mathrm{~g} \mathrm{~L}^{-1}$ ) with or without 5-fluoroorotic acid (5-FOA) was used to screen transformants generated during the genetic manipulation of $C$. tropicalis XZX. Fermentation medium $\left(5 \mathrm{~g} \mathrm{~L}^{-1}\right.$ yeast extract containing xylose and/or glucose at the indicated concentration) and XML medium $\left(5 \mathrm{~g} \mathrm{~L}^{-1}\right.$ yeast extract containing XML at the indicated concentration) were used to evaluate the xylitol production performance of engineered strains. E. coli JM109 (TaKaRa, Dalian, China), which was used as the host for all genetic manipulations, was cultured at $37^{\circ} \mathrm{C}$ in $\mathrm{LB}$ medium $\left(5 \mathrm{~g} \mathrm{~L}^{-1}\right.$ yeast extract, $10 \mathrm{~g} \mathrm{~L}^{-1}$ peptone, $10 \mathrm{~g} \mathrm{~L}^{-1} \mathrm{NaCl}$ ) supplemented with $100 \mu \mathrm{g} \mathrm{mL}^{-1}$ ampicillin. Solid media were prepared by adding agar to a final concentration of $20 \mathrm{~g} \mathrm{~L}^{-1}$. The XML used in this study was supplied by Futian Pharmaceutical Co., Ltd. (Dezhou, Shandong Province, China).

\section{Genetic manipulations}

Deletion of XYL2. The XYL2 gene (GenBank accession number: DQ201637.1) was sequentially deleted using the method described in our previous work [19]. The procedure is outlined in Additional file 1: Fig. S7. Firstly, the XYL2 gene was amplified from $C$. tropicalis XZX genomic DNA using PCR using primers UXYL2 and DXYL2 (Table 3), and ligated into the plasmid vector pMD 18-T Simple (TaKaRa, Dalian, China) to yield plasmid Ts-XYL2. Then, inverse PCR with primers rUXYL2 and rDXYL2 (Table 3) was used to eliminate the middle region of $X Y L 2$ (approximately $700 \mathrm{bp}$ ). The resulting PCR product (XYL2I-Ts-XYL2I) was digested with PstI and $X b a \mathrm{I}$ and ligated to gda324-URA3 cassettes

Table 3 Primers used in this study

\begin{tabular}{|c|c|c|}
\hline Primers & Sequence ${ }^{a}\left(5^{\prime}\right.$ to $\left.3^{\prime}\right)$ & Restriction sites \\
\hline UXYL2 & TAAATAGAACCCACGAATCCCT & \\
\hline DXYL2 & TTTACTCGTACTATGCACTCC & \\
\hline rUXYL2 & $\begin{array}{l}\text { AACTGCAGAGTAGTGAATATCGGAAC } \\
\text { CACA }\end{array}$ & Pstl \\
\hline rDXYL2 & GCTCTAGAAACTTCCCAATTTCCGACT & Xbal \\
\hline U2XYL2 & CTAAATCCGGCCACTACCAC & \\
\hline D2XYL2 & CCAGCGTTACCAATTTGCAC & \\
\hline rU2XYL2 & AACTGCAGTCACCGAACTTCAAATCAGC & Pstl \\
\hline rD2XYL2 & GCICTAGATGCCGTTGCCAGAACCAT & Xbal \\
\hline UPGAPDH & CGGGATCCAACGTGGTATGGTTGT & Xhol \\
\hline DPGAPDH & $\underset{\text { GGAT }}{\text { GCTAGATGTTTAAATTCTTTAATTGAG }}$ & BamHI \\
\hline UTGAPDH & CGACGCGTCTATCCAACAAACT & Xbal \\
\hline DTGAPDH & CCGCTCGAGTCTGGTTTAGAAGTAGG & Mlul \\
\hline UZWF & GCICTAGAATGACTGGCACCTTACCC & $X b a l$ \\
\hline DZWF & CGACGCGTCACGAGGAGCCCTT & $X b a l$ \\
\hline UGND & GCTCTAGAATGACTGACACTTCAAAC & Mlul \\
\hline DGND & CGACGCGTTTAAGCATCGT & Xbal \\
\hline UMAE & GCGTCGACACCCGATTTCAAAAGTGCAGA & Mlul \\
\hline DMAE & CGACGCGTCTAGTCGTAATCCC & Xbal \\
\hline YIZWF-F & AAGAACACCATTTCCAAC & For RT-qPCR \\
\hline YIZWF-R & GATGATTCCAATGTCGTT & For RT-qPCR \\
\hline YIGND-F & CCAACACGAATACTAACA & For RT-qPCR \\
\hline YIGND-R & TTAACGAGCAGAATGATT & For RT-qPCR \\
\hline YIMAE-F & AACGAGGTGCTCTACTAC & For RT-qPCR \\
\hline YIMAE-R & CTGAGTCGGTGTATAGATGAT & For RT-qPCR \\
\hline
\end{tabular}

${ }^{a}$ Restriction sites introduced into the primers are underlined 
(containing a functional yeast $U R A 3$ gene flanked by a $324 \mathrm{bp}$ gene disruption auxiliary sequence ( $g$ da324)) yielding plasmid Ts-XYL2I-gda-URA3. The disruption cassette for the sequential removal of both $X Y L 2$ alleles was isolated from plasmid Ts-XYL2I-gda-URA3 using PCR with primers UXYL2 and DXYL2. An established lithium chloride method [19] was used to transform C. tropicalis XZX with this cassette. Correct transformants were identified on MM plates, and confirmed by PCR and DNA sequencing. After removal of the URA3 marker, the resulting strain was used for disruption of the second $X Y L 2$ allele. A similar method was used to disrupt the second $X Y L 2$ allele, generating the mutant strain $C$. tropicalis XZX-B4 ( $x l y 2 / x y l 2)$.

Construction of coenzyme regeneration systems in C. tropicalis $X Z X-B 4$. The promoter and terminator of the glyceraldehyde-3-phosphate dehydrogenase gene (GAPDH, GenBank accession number: HQ171163.1) were amplified from the C. tropicalis ATCC 20,336 genome using PCR with primers UPGAPDH and DPGAPDH, and UTGAPDH and DTGAPDH, respectively (Table 2). The terminator fragment was inserted into pMD 18-T (TaKaRa, Dalian, China) to generate plasmid Tm- $T_{\text {GAPDH }}$. The promoter fragment was digested with $X b a \mathrm{I}$ and $B a m \mathrm{HI}$, and inserted into similarly digested Tm- $T_{G A P D H}$ to generate plasmid Tm- $P_{G A P D H^{-}}$ $T_{\text {GAPDH. }}$. The open reading frame of the 6-phosphateglucose dehydrogenase gene ( $Y l Z W F$, GenBank accession number: CP017557.1) from Yarrowia lipolytica CICC 31,251 was amplified using PCR with primers UZWF and DZWF and then inserted into Tm- $P_{\text {GAPDH }}-T_{\text {GAPDH }}$ between its $M l u \mathrm{I}$ and $X b a \mathrm{I}$ sites to generate plasmid Tm- $P_{\text {GAPDH }}-Y l Z W F-T_{G A P D H}$. This plasmid was digested with HindIII and BamHI to generate the $P_{\text {GAPDH }}-Y l Z W F$ $T_{G A P D H}$ cassette. After blunting its sticky ends using $p f u$ DNA polymerase (BBI, Shanghai, China), the cassette was inserted into plasmid Ts-XYL2I-gda-URA3 that had been digested with $X b a \mathrm{I}$ and had its sticky ends blunted, yielding plasmid Ts-XYL2I-gda-URA3- $P_{G A P D H}-Y l Z W F-$ $T_{\text {GAPDH. }}$ A $6.1 \mathrm{~kb} Y l Z W F$ expression cassette (XYL2Igda-URA3-P GAPDH $\left.^{-} Y l Z W F-T_{\text {GAPDH }}-X Y L 2 I\right)$ was obtained using PCR with primers UXYL2 and DXYL2. This cassette was inserted into C. tropicalis XZX-B4 using the lithium chloride method (the resulting strain was designated as C. tropicalis XZX-B4Z), and then the marker gene was removed to produce the strain $C$. tropicalis XZX-B4Zt.

A similar method was used to construct Ts-XYL2IIgda-URA3- $P_{G A P D H^{-}} Y l G N D-T_{G A P D H}$, a plasmid containing an expression cassette for the 6-phosphate-gluconic acid dehydrogenase gene (YlGND, GenBank accession number: CP017554.1) from $Y$. lipolytica. In short, the YlGND open reading frame was amplified from $Y$. lipolytica CICC 31,251 using PCR with primers UGND and DGND. The resulting PCR fragment was inserted between the $M l u \mathrm{I}$ and $X b a \mathrm{I}$ sites of Tm- $P_{G A P D H}-T_{G A P D H}$ to obtain plasmid Tm- $P_{G A P D H^{-}} Y l G N D-T_{G A P D H}$. This plasmid was digested with HindIII and EcoRI to obtain the $P_{G A P D H^{-}} Y l G N D-T_{G A P D H}$ cassette, which was inserted into the XbaI site of plasmid Ts-XYL2II-gda-URA3 using a blunt-end ligation technique. This plasmid was designated Ts-XYL2II-gda-URA3-P $P_{G A P D H^{-}} Y l G N D-T_{G A P D H}$. The YlGND expression cassette XYL2II-gda-URA3-P ${ }_{G A P D H^{-}}$ $Y l G N D-T_{G A P D H^{-}} X Y L 2 I I$, which was obtained using PCR with primers U2XYL2 and D2XYL2, was inserted into $C$. tropicalis XZX-B4 and XZX-B4Zt using the lithium chloride method, respectively. This process generated strain C. tropicalis XZX-B4G and XZX-B4ZG.

The YlMAE open reading frame (GenBank accession number: XM-504112.1), which encodes the malic enzyme, was amplified from Y. lipolytica CICC 31,251 genomic DNA using PCR with primers UMAE and DMAE. The YIMAE expression cassette XYL2I-gdaURA3- $P_{G A P D H^{-}} Y l M A E-T_{G A P D H}-X Y L 2 I$ was constructed using the method described above (see Additional file 1: Fig. S3a). Finally, the cassette was used to transform $C$. tropicalis XZX-B4, generating C. tropicalis XZX-B4M.

\section{Shake-flask experiments}

To assess $X Y L 2$ gene function and the NADPH regeneration systems, strains were pre-cultured for $48 \mathrm{~h}$ in 100$\mathrm{mL}$ shake flasks containing $20 \mathrm{~mL}$ of SM medium. These cultures were used to inoculate SM (glucose or xylose as a sole carbon source). Cell density was determined using the absorbance of the culture at $600 \mathrm{~nm}\left(\mathrm{OD}_{600}\right)$, which was measured with a spectrophotometer. Concentrations of xylose and xylitol were determined using HPLC.

For xylitol production, strains were initially cultured for $48 \mathrm{~h}$ in YPD medium $\left(10 \mathrm{~g} \mathrm{~L}^{-1}\right.$ yeast extract, $20 \mathrm{~g}$ $\mathrm{L}^{-1}$ peptone, $20 \mathrm{~g} \mathrm{~L}^{-1}$ glucose) at $30{ }^{\circ} \mathrm{C}$ and $200 \mathrm{rpm}$. These seed cultures were used to inoculate 250 -mL flasks containing $100 \mathrm{~mL}$ of fermentation medium or XML medium to the indicated starting $\mathrm{OD}_{600}$ value. During fermentation, the concentrations of xylose and xylitol and the cell density were determined at appropriate intervals.

\section{Bioreactor experiments}

A single colony of $C$. tropicalis XZX-B4ZG was used to inoculate $20 \mathrm{~mL}$ of YPD medium in a $100-\mathrm{mL}$ shake flask. The resulting culture was incubated on a rotary shaker at $30{ }^{\circ} \mathrm{C}$ and $200 \mathrm{rpm}$. This seed culture was used to inoculate a $250-\mathrm{mL}$ shake flask containing $50 \mathrm{~mL}$ of optimized seed medium (YPD supplemented with $200 \mathrm{~g} \mathrm{~L}^{-1}$ $\mathrm{XML}$ ). After incubation for $70 \mathrm{~h}$ at $30{ }^{\circ} \mathrm{C}$ and $200 \mathrm{rpm}$, about $20 \%(\mathrm{v} / \mathrm{v})$ of this seed culture were transferred into a 5-L bioreactor (Bailun Co., China) containing $2.5 \mathrm{~L}$ of 
fermentation medium supplemented with $5 \mathrm{~g} \mathrm{~L}^{-1}$ corn steep liquor and $300 \mathrm{~g} \mathrm{~L}^{-1} \mathrm{XML}$. The initial $\mathrm{pH}$ was 5.0, and the $\mathrm{pH}$ during the fermentation was not controlled. Fermentations for xylitol production were performed at $35{ }^{\circ} \mathrm{C}$ and $700 \mathrm{rpm}$, and air was supplied at $4 \mathrm{vvm}$. In each run, glucose was added at a final concentration of $5 \mathrm{~g} \mathrm{~L}^{-1}$ at 29,50 and $79 \mathrm{~h}, 3 \mathrm{~g} \mathrm{~L}^{-1}$ at $100 \mathrm{~h}$ to the bioreactor (glucose concentration lower than $1 \mathrm{~g} \mathrm{~L}^{-1}$ ). Samples were collected at appropriate intervals and the concentrations of residual sugars and xylitol were determined using HPLC.

\section{RNA and cofactor levels}

Relative YlZWF, YlGND and YlMAE messenger RNA (mRNA) levels in $C$. tropicalis were measured using real-time quantitative PCR (RT-qPCR) as previously described [19]. The intracellular of NADPH and NADP ${ }^{+}$ concentrations were measured using the NADP/NADPH Quantification Colorimetric Kit (Bio Vision, Bay Area, USA) as recommended by the manufacturer. Yeast cells were grown in SM and harvested during the appropriate growth period.

\section{Analytical methods}

To quantify the concentrations of furfural, D-glucose, L-arabinose, D-xylose and xylitol, diluted supernatants were analyzed using an Agilent 1260 HPLC system (Agilent Technologies Inc., Santa Clara, CA) equipped with refractive index detector at $35{ }^{\circ} \mathrm{C}$ and an Aminex HPX$87 \mathrm{H}$ cation exchange column $(300 \mathrm{~mm} \times 7.8 \mathrm{~mm})$. The column was eluted with $5 \mathrm{mM} \mathrm{H}_{2} \mathrm{SO}_{4}$ (flow rate $0.5 \mathrm{~mL} \mathrm{~min}^{-1}$ ) at $50{ }^{\circ} \mathrm{C}$ temperature. Before use, the mobile phase was purified by vacuum filtration through a $0.22 \mu \mathrm{m}$ microfiltration membrane and trapped air was removed using an ultrasonic cleaner. Prior to analysis, $1 \mathrm{~mL}$ of the fermentation broth or XML was centrifuged at $10,000 \mathrm{~g}$ for $5 \mathrm{~min}$, and the supernatant were filtered through a $0.22 \mu \mathrm{m}$ pore diameter cellulose acetate membrane. The concentration of acetate, furfural and 5 -hydroxymethyl furfural in XML was determined as previously reported $[1,18,26]$.

\section{Statistical analysis}

Data are presented as means $\pm \mathrm{SD}$. The statistically significance of differences was analyzed using Student's $t$ test. Differences resulting in values of $P<0.05$ were considered significant.

\section{Supplementary Information}

The online version contains supplementary material available at https://doi. org/10.1186/s12934-021-01596-1.

Additional file 1: Figure S1. Growth curve of $C$. tropicalis XZX in XML medium. A colony of XZX was pre-culture in YPD medium, and diluted to a density of 0.5 at $600 \mathrm{~nm}\left(\mathrm{OD}_{600}\right)$ in $250 \mathrm{~mL}$ shake flasks with $50 \mathrm{~mL}$ XML medium ( $5 \mathrm{~g} \cdot \mathrm{L}^{-1}$ yeast extract, $200 \mathrm{~g} \cdot \mathrm{L}^{-1} \mathrm{XML}$ ) at $200 \mathrm{rpm}$ and 30 ${ }^{\circ} \mathrm{C}$. Figure S2. Confirmation of growth phenotype using xyloseas the sole carbon source. XZX, C.tropicalis XZX (parent strain); XZX-B2, XZX with one $X Y L 2$ allele deleted; $X Z X-B 4, X Z X$ with both XYL2 alleles deleted. Figure $\mathbf{S 3}$. Verification of $C$. tropicalis mutants using PCR. (a) Structure of integration cassette used to overexpress YIMAE. Similar cassettes were used to overexpress YIZWF and YIGND. (b) Verification of XZX-B4 and XZX-B4Zt by PCR using primers UXYL2 and UXYL2.M1: DL15 000 DNA marker; M2: DL5 000 DNA marker; lane 1:XZX-B4; lane 2: XZX-B4; (c) Verification of XZX-B4ZG by PCR. M1: DL15 000 DNA marker; M2: DL5 000 DNA marker; lane 1: XZX B4Z; lane 2: XZX B4; (d) Verification of XZX-B4M by PCR. M1: DL15 000 DNA marker; M2: DL5 000 DNA marker; lane 1: XZX B4; lane 2: XZX-B4M. Figure S4. Evaluation of YIZWF, YIGND and YIMAE expression using qPCR. Evaluation of: (a) YIZWF expression by two transformants of C. tropicalis XZX-B4Z, compared with control strain XZX; (b) YIGND expression by two transformants of C. tropicalis XZX-B4ZG, compared with control strain XZX; and (c) YIMAE expression by two transformants of $C$. tropicalis XZX-B4M,compared with control strain XZX; Error bars indicate standard deviations from the means of triplicate biological replicates. Figure S5. Growth curve of XZX-B3, XZX-B4Z and XZX-B4ZG in fermentation medium $\left(6.7 \mathrm{~g} \cdot \mathrm{L}^{-1}\right.$ yeast nitrogen base, $10 \mathrm{~g} \cdot \mathrm{L}^{-1}$ glucose, $50 \mathrm{~g} \cdot \mathrm{L}^{-1}$ xylose, $10 \mathrm{~g} \cdot \mathrm{L}^{-1}\left(\mathrm{NH}_{4}\right)_{2} \mathrm{SO}_{4}, 0.06$ $\mathrm{g} \cdot \mathrm{L}^{-1}$ uracil and $2 \mathrm{~g} \cdot \mathrm{L}^{-1}$ furfural). Figure $\mathrm{S} 6$. Effect of nitrogen sources on xylitol production by C. tropicalis XZX-B4ZG in shake flasks. The experiment was done in $250 \mathrm{~mL}$ shake flasks with $100 \mathrm{~mL}$ fermentation medium (250 g. $\cdot \mathrm{L}^{-1} \mathrm{XML}$ and $5 \mathrm{~g} \cdot \mathrm{L}^{-1}$ nitrogen sources, $\mathrm{pH}$ 5.0) at $200 \mathrm{rpm}$ and $35^{\circ} \mathrm{C}$. (a): Effect on cell growth (b): Effect on xylose concentration (c) Effect on xylitol concentration. Figure S7. XYL2 deletion in C. tropicalis XZX. Schematic depiction of the sequential disruption of the two XYL2 alleles in C. tropicalis using miniature disruption cassettes. Table S1. Performance of fermentations using $100 \mathrm{~g} \cdot \mathrm{L}^{-1} \mathrm{XML}$ as substrate.

\section{Acknowledgements}

We thank Haiquan Yang, Yuanyuan Xia and Lei Chen at Jiangnan University for their assistance.

\section{Authors' contributions}

$X C$ and QL conceived and designed research. $L Z, Z C$ and JW performed the research. LZ, WS and XC analyzed data. LZ and XC wrote the manuscript. All authors read and approved the manuscript.

\section{Funding}

This work was supported by Natural Science Foundation of Jiangsu province (No. BK20171138), the 111 Project (No. 111-2-06), Top-notch Academic Programs Project of Jiangsu Higher Education Institutions and Postgraduate Research and Practice Innovation Program of Jiangsu Province (KYCX20-1807).

Availability of data and materials

All data generated or analyzed during this study are included in this published article and its supplementary information files.

\section{Declarations}

Ethics approval and consent to participate Not applicable.

\section{Consent for publication}

Not applicable.

\section{Competing interests}

The authors declare that they have no conflict of interests. 
Received: 22 March 2021 Accepted: 15 May 2021

Published online: 25 May 2021

\section{References}

1. Wang H, Li L, Zhang L, An J, Cheng H, Deng Z. Xylitol production from waste xylose mother liquor containing miscellaneous sugars and inhibitors: one-pot biotransformation by Candida tropicalis and recombinant Bacillus subtilis. Microb Cell Fact. 2016;15:82.

2. Li Z, Guo XX, Feng XD, Li C. An environment friendly and efficient process for xylitol bioconversion from enzymatic corncob hydrolysate by adapted Candida tropicalis. Chem Eng J. 2015;263:249-56.

3. Li ZL, Xiao H, Jiang WH, Jiang Y, Yang S. Improvement of solvent production from xylose mother liquor by engineering the xylose metabolic pathway in Clostridium acetobutylicum EA 2018. Appl Biochem Biotech. 2013;171:555-68.

4. Venkateswar Rao L, Goli JK, Gentela J, Koti S. Bioconversion of lignocellulosic biomass to xylitol: an overview. Bioresour Technol. 2016:213:299-310.

5. Cheng HR, Wang B, LV JY, Jiang MG, Lin SJ, Deng ZX. Xylitol production from xylose mother liquor: a novel strategy that combines the use of recombinant Bacillus subtilis and Candida maltosa. Microb Cell Fact. 2011:10:2.

6. Jin LQ, Yang B, Xu W, Chen XX, Jia DX, Liu ZQ, Zheng YG. Immobilization of recombinant Escherichia coli whole cells harboring xylose reductase and glucose dehydrogenase for xylitol production from xylose mother liquor. Bioresour Technol. 2019:285:121344.

7. Wang HH, Pan JC, Wang J, Wang N, Zhang J, Li Q, et al. Succinic acid production from xylose mother liquor by recombinant Escherichia coli strain. Biotechnol Biotec Eqs. 2014:28:1042-1049.

8. Cheng $H$, Wang $H$, LV J, Jiang M, Lin S, Deng Z. A novel method to prepare $L$-arabinose from xylose mother liquor by yeast-mediated biopurification. Microb Cell Fact. 2011;10:43.

9. Xiao H, Li Z, Jiang Y, Yang Y, Jiang W, Gu Y, Yang S. Metabolic engineering of D-xylose pathway in Clostridium beijerinckii to optimize solvent production from xylose mother liquid. Metab Eng. 2012;14:569-78.

10. Liu X, Lin L, Xu X, Zhang H, Wu L, Zhu P, et al. Two-step economical welan gum production by Sphingomonas sp. HT-1 from sugar industrial byproducts. Carbohydr Polym. 2018;181:412-8.

11. Allen SA, Clark W, McCaffery JM, Cai Z, Lanctot A, Slininger PJ, et al. Furfural induces reactive oxygen species accumulation and cellular damage in Saccharomyces cerevisiae. Biotechnol Biofuels. 2010;3:1754-68.

12. Zaldivar J, Martinez A, Ingram LO. Effect of alcohol compounds found in hemicellulose hydrolysate on the growth and fermentation of ethanologenic Escherichia coli. Biotechnol Bioeng. 2000:68:524-30.

13. Ping $Y$, Ling $H Z$, Song G, Ge JP. Xylitol production from non-detoxified corncob hemicellulose acid hydrolysate by Candida tropicalis. Biochem Eng J. 2013;75:86-91.

14. Kim TB, Oh DK. Xylitol production by Candida tropicalis in a chemically defined medium. Biotechnol Lett. 2003:25:2085-8.

15. Ko BS, Kim J, Kim JH. Production of xylitol from D-xylose by a xylitol dehydrogenase gene-disrupted mutant of Candida tropicalis. Appl Environ Microb. 2006;72:4207-13.

16. Ahmad I, Shim WY, Jeon WY, Yoon BH, Kim JH. Enhancement of xylitol production in Candida tropicalis by co-expression of two genes involved in pentose phosphate pathway. Bioproc Biosyst Eng. 2012;35:199-204.

17. Ahmad I, Shim WY, Kim JH. Enhancement of xylitol production in glycerol kinase disrupted Candida tropicalis by co-expression of three genes involved in glycerol metabolic pathway. Bioproc Biosyst Eng. 2013;36:1279-84.

18. Wang SZ, Cheng G, Joshua C, He ZJ, Sun XX, Li RM, et al. Furfural tolerance and detoxification mechanism in Candida tropicalis. Biotechnol Biofuels. 2016:9:250

19. Zhang $L H$, Chen $X Z$, Chen Z, Wang ZZ, Jiang $S$, Li L, et al. Development of an efficient genetic manipulation strategy for sequential gene disruption and expression of different heterologous GFP genes in Candida tropicalis. Appl Microbiol Biot. 2016:100:9567-80.

20. Chen Z, Chen XZ, Zhang LH, Wang JH, Shen W, Fan Y. Metabolic engineering of Candida tropicalis for xyltiol production from xylose mother liquor. Chin J Biotechnol. 2017:37(5):66-75.

21. Verho R, Londesborough J, Penttila M, Richard P. Engineering redox cofactor regeneration for improved pentose fermentation in Saccharomyces cerevisiae. Appl Environ Microbiol. 2003;69:5892-7.

22. Liu W, Wang P. Cofactor regeneration for sustainable enzymatic biosynthesis. Biotechnol Adv. 2007;25:369-84.

23. Chemler JA, Fowler ZL, McHugh KP, Koffas MA. Improving NADPH availability for natural product biosynthesis in Escherichia coli by metabolic engineering. Metab Eng. 2010:12:96-104.

24. Moreira dos Santos M, Raghevendran V, Kotter P, Olsson L, Nielsen J. Manipulation of malic enzyme in Saccharomyces cerevisiae for increasing NADPH production capacity aerobically in different cellular compartments. Metab Eng. 2004;6:352-63.

25. Gorsich SW, Dien BS, Nichols NN, Slininger PJ, Liu ZL, et al. Tolerance to furfural-induced stress is associated with pentose phosphate pathway genes ZWF1, GND1, RPE1, and TKL1 in Saccharomyces cerevisiae. Appl Microbiol Biot. 2006;71:339-349.

26. Chen XZ, Tian KM, Niu DD, Shen W, Algasan G, Singh S, et al. Efficient bioconversion of crude glycerol from biodiesel to optically pure D-lactate by metabolically engineered Escherichia coli. Green Chem. 2014:16:342-50.

27. Carmel-Harel O, Storz G. Roles of the glutathione- and thioredoxindependent reduction systems in the Escherichia coli and Saccharomyces cerevisiae responses to oxidative stress. Annu Rev Microbiol. 2000:54:439-61.

28. Hedeskov CJ, Capito K, Thams P. Cytosolic ratios of free [NADPH]/[NADP $\left.{ }^{+}\right]$ and $[\mathrm{NADH}] /\left[\mathrm{NAD}^{+}\right]$in mouse pancreatic islets, and nutrient-induced insulin secretion. Biochem J. 1987:241:161-7.

29. Hua Y, Wang J, Zhu Y, Zhang B, Kong X, Li W, et al. Release of glucose repression on xylose utilization in Kluyveromyces marxianus to enhance glucose-xylose co-utilization and xylitol production from corncob hydrolysate. Microb Cell Fact. 2019;18:24.

30. Hua Y, Wang J, Zhu Y, Zhang B, Kong X, Li W, Wang D, Hong J. Release of glucose repression on xylose utilization in Kluyveromyces marxianus to enhance glucose-xylose co-utilization and xylitol production from corncob hydrolysate. Microb Cell Fact. 2019;18:24.

31. Xu Y, Chi P, Bilal M, Cheng H. Biosynthetic strategies to produce xylitol: an economical venture. Appl Microbiol Biot. 2019;103:5143-60.

32. Yoon BH, Jeon WY, Shim WY, Kim JH. L-arabinose pathway engineering for arabitol-free xylitol production in Candida tropicalis. Biotechnol Lett. 2011:33:747-53.

\section{Publisher's Note}

Springer Nature remains neutral with regard to jurisdictional claims in published maps and institutional affiliations. 